\title{
Genome-wide transcriptome analysis reveals that a pleiotropic antibiotic regulator, AfsS, modulates nutritional stress response in Streptomyces coelicolor A3(2)
}

\author{
Wei Lian $^{\dagger 1}$, Karthik P Jayapal ${ }^{\dagger 1}$, Salim Charaniya1, Sarika Mehra1, \\ Frank Glod², Yun-Seung Kyung1, David H Sherman² and Wei-Shou Hu*1
}

Address: ${ }^{1}$ Department of Chemical Engineering and Materials Science, University of Minnesota, 421 Washington Ave. SE., Minneapolis, MN 55455, USA and 2Life Sciences Institute, Departments of Medicinal Chemistry, Chemistry, Microbiology \& Immunology, University of Michigan, 210 Washtenaw Ave., Ann Arbor, MI 48109, USA

Email: Wei Lian - wei.lian@abbott.com; Karthik P Jayapal - jayapal@cems.umn.edu; Salim Charaniya - salim@cems.umn.edu; Sarika Mehra - sarika@che.iitb.ac.in; Frank Glod - Frank.GLOD@fnr.lu; Yun-Seung Kyung - ykyung@cntus.jnj.com;

David H Sherman - davidhs@lsi.umich.edu; Wei-Shou Hu* - acre@cems.umn.edu

* Corresponding author †Equal contributors

Published: 29 January 2008

BMC Genomics 2008, 9:56 doi:10.1186/147/-2164-9-56

This article is available from: http://www.biomedcentral.com/I47I-2/64/9/56

(c) 2008 Lian et al; licensee BioMed Central Ltd.

This is an Open Access article distributed under the terms of the Creative Commons Attribution License (http://creativecommons.org/licenses/by/2.0), which permits unrestricted use, distribution, and reproduction in any medium, provided the original work is properly cited.

\begin{abstract}
Background: A small "sigma-like" protein, AfsS, pleiotropically regulates antibiotic biosynthesis in Streptomyces coelicolor. Overexpression of afsS in S. coelicolor and certain related species causes antibiotic stimulatory effects in the host organism. Although recent studies have uncovered some of the upstream events activating this gene, the mechanisms through which this signal is relayed downstream leading to the eventual induction of antibiotic pathways remain unclear.
\end{abstract}

Results: In this study, we employed whole-genome DNA microarrays and quantitative PCRs to examine the transcriptome of an afs disruption mutant that is completely deficient in the production of actinorhodin, a major S. coelicolor antibiotic. The production of undecylprodigiosin, another prominent antibiotic, was, however, perturbed only marginally in the mutant. Principal component analysis of temporal gene expression profiles identified two major gene classes each exhibiting a distinct coordinate differential expression pattern. Surprisingly, nearly $70 \%$ of the $>117$ differentially expressed genes were conspicuously associated with nutrient starvation response, particularly those of phosphate, nitrogen and sulfate. Furthermore, expression profiles of some transcriptional regulators including at least two sigma factors were perturbed in the mutant. In almost every case, the effect of afsS disruption was not observed until the onset of stationary phase.

Conclusion: Our data suggests a comprehensive role for S. coelicolor Afs $\mathrm{S}$ as a master regulator of both antibiotic synthesis and nutritional stress response, reminiscent of alternative sigma factors found in several bacteria.

\section{Background}

Streptomycetes are common saprophytic soil bacteria that constitute some of the most proficient producers of natu- rally occurring therapeutic molecules like antibiotics, immunosuppressants and anti-cancer agents [1]. The regulation of biosynthesis of these compounds has therefore 
evoked considerable interest among researchers. It is wellknown that antibiotic biosynthesis in bacteria is generally elicited as a physiological response to a variety of environmental stimuli including high cell density, nutritional imbalance and/or presence of stress-inducing agents. In streptomycetes, evidence hinting at the interplay between stress signals and antibiotic synthesis have emerged with the findings that mutants of several Streptomyces coelicolor sigma factors implicated in stress-response are also perturbed in antibiotics production [2-8]. Growth limitations resulting from nutritional imbalances like nitrogen or phosphate starvation are also known to trigger antibiotic synthesis $[9,10]$. Yet, despite extensive efforts aimed at elucidating antibiotic regulatory pathways, the exact chain of molecular events leading from sensing of stress or nutritional starvation signal to the eventual activation of antibiotic pathways remains largely obscure.

Years of genetic research, recently aided by the availability of complete genome sequence of $S$. coelicolor [11], has identified several key players involved in the regulation of secondary metabolism in $S$. coelicolor. One such set of genes is the AfsK-AfsR-AfsS system that globally controls antibiotic synthesis under certain conditions (reviewed in $[12,13])$. When AfsK, one of numerous serine/threonine kinases in S. coelicolor, is autophosphorylated, it phosphorylates AfsR. Phosphorylated AfsR has enhanced DNAbinding activity specific to the promoter of $a f_{s} S$ and positively regulates its transcription. AfsS is then proposed to lead to the onset of antibiotic production in an as yet unknown manner. Recent studies have uncovered some of the upstream events leading to the activation of this signaling pathway. It has now been proposed that the autophosphorylation of AfsK is caused by increased levels of $S$ adenosyl- ${ }_{\mathrm{L}}$-methionine, a probable intracellular signaling factor [14], and that this phosphorylation is modulated by another protein, KbpA [15]. In addition, phosphorylation of AfsR by other serine/threonine kinases like PkaG and AfsL have also been demonstrated [16]. An understanding of the signaling cascade downstream of the AfsKAfsR-AfsS system, however, continues to remain elusive.

In this study we employ DNA microarrays to perform a genome-wide transcriptome profiling of a $S$. coelicolor afs $S$ disruption mutant. Overexpression of afs $S$ in $S$. coelicolor and S. lividans had previously been shown to significantly enhance actinorhodin synthesis $[17,18]$ while disruption of afsS diminished actinorhodin production [19]. We show here that the regulatory implications of AfsS are not limited to modulation of antibiotic synthesis but extend to the control of certain phosphate starvation response, and nitrogen and sulfate metabolism genes as well. This suggests a far more pleiotropic role for AfsS than that theorized by our current understanding and provides an important link between nutritional stress and activation of antibiotic pathways in S. coelicolor. Furthermore, clustering and comparative transcriptome analysis of wildtype and this mutant enabled us to gain insights into the biological functions of certain poorly annotated genes.

\section{Results and Discussion \\ Construction of an afsS mutant}

The gene afsS encodes a relatively small 63-amino-acid "sigma-like" protein containing three repeats of a short 12-amino acid segment that are thought to be crucial for its activity [19]. To investigate the physiological role of AfsS in S. coelicolor M145, a disruption mutant was constructed by replacing two of these tandem repeats with an apramycin resistance cassette. The deleted segment was 104 bp in length (+26 to +129 from the translational start site) and the resulting strain was designated as YSK4425.

\section{Growth and antibiotic production kinetics}

Figure 1a-c shows the time profiles of growth and antibiotic titers of M145 and YSK4425 measured during the course of a two-day liquid culture in R5-medium. Disruption of afs $S$ did not significantly change the growth kinetics. However, synthesis profiles of two major pigmented antibiotics were altered in the mutant. The most significant change was observed for the polyketide antibiotic, actinorhodin, which normally accumulates to significant levels in M145 giving the culture a distinctive deep blue color. Production of this antibiotic was completely abolished in YSK4425 (Figure 1c and 1d). This finding is much more dramatic than earlier reports where synthesis of actinorhodin was observed in afsS deletion strains [19], albeit to a much lesser extent compared to wild-type. This difference was most likely due to our use of R5- medium [20] which does not contain any inorganic phosphate source. Significant actinorhodin accumulation was observed in YSK4425 cultures grown in phosphate containing R2YE medium. Inorganic phosphate depletion is an important trigger for antibiotic synthesis in S. coelicolor [21] and, as will be shown later, AfsS is a possible link between phosphate starvation response and actinorhodin synthesis. Another major antibiotic in S. coelicolor, a redpigmented tripyrrole, undecylprodigiosin, accumulated to a slightly lower extent in YSK4425 mainly in stationary phase.

These observations were confirmed by performing an independent biological replicate culture. Genetic complementation of afsS in YSK4425 restored antibiotic synthesis (Figure 1d). Taken together, these findings reaffirm the pleiotropic role of afsS in regulation of antibiotic synthesis in S. coelicolor.

\section{Microarray analysis}

Whole-genome microarrays containing duplicate probes for $\sim 96 \%$ of the predicted ORFs in S. coelicolor were fabri- 
a.

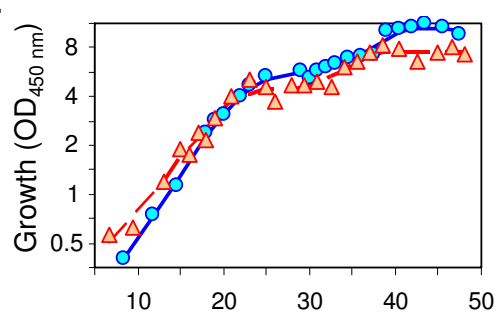

b.

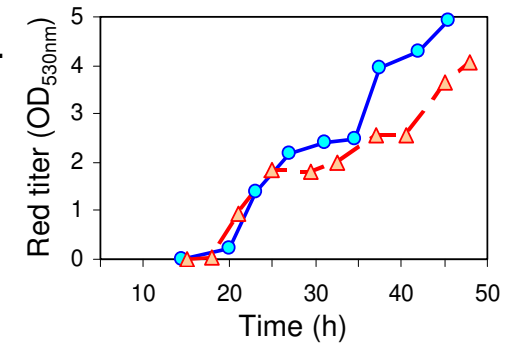

c.

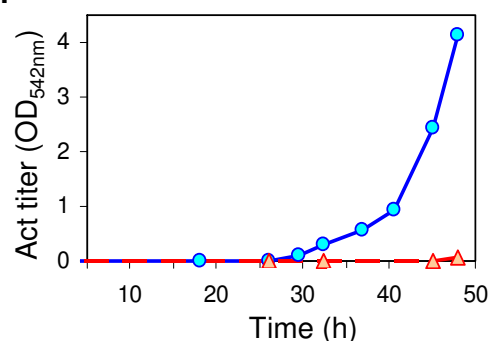

d.

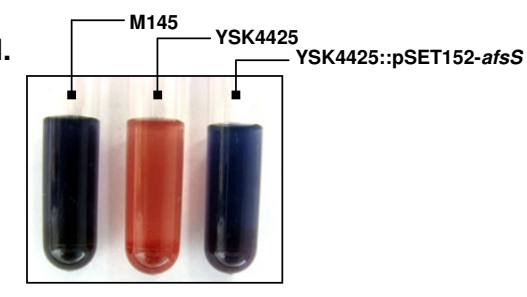

Figure I

Growth and antibiotic synthesis kinetics of MI45 and YSK4425. (a) Growth measured using optical density at $450 \mathrm{~nm}$. (b) and (c) Spectrophotometric measurements of antibiotic titers for undecylprodigiosin and actinorhodin. Time profiles for MI45 (O, solid blue line) and YSK4425 ( $\triangle$, dashed red line) are shown. The curves represent data from one of two reproducible experiments. (d) Photograph shows the dramatic difference in antibiotic synthesis between the MI45 and YSK4425. Pictures are culture samples taken $\sim 4$ days after inoculation, indicating that the observed absence of actinorhodin synthesis was a genuine abolishment rather than a delay in synthesis.

cated as reported earlier [22]. Fluorescently labeled cDNA prepared from total RNA extracts isolated during various growth stages were used in hybridizations. The sampling points chosen for M145 were $15 \mathrm{~h}, 18 \mathrm{~h}, 19 \mathrm{~h}, 21 \mathrm{~h}, 23 \mathrm{~h}$, $25 \mathrm{~h}, 27 \mathrm{~h}, 29 \mathrm{~h}, 32 \mathrm{~h}, 34 \mathrm{~h}, 37 \mathrm{~h}, 39 \mathrm{~h}$ and $42 \mathrm{~h}$; for YSK4425 15 h, 17 h, 19 h, 21 h, 23 h, 25 h, 28 h, 31 h, 32 $\mathrm{h}, 37 \mathrm{~h}, 38 \mathrm{~h}, 41 \mathrm{~h}$ and $45 \mathrm{~h}$ were chosen. Genomic DNA (gDNA) was used as a universal reference for all hybridizations. The relative fluorescence intensity of cDNA to gDNA, referred to hereafter as the ' $\log _{2}$ expression value', is thus an estimate of transcript abundance. Hybridizations were generally performed as duplicates and outlier data points from four spots (duplicate spots from duplicate chips) were filtered out using a mean \pm 1.2 times standard deviation cut-off. Key conclusions reported here were confirmed by additional hybridizations performed on the biological replicate culture.

Identification of kinetically perturbed genes in afsS mutant After normalization, the overall $\log _{2}$ expression values for both M145 and YSK4425 samples ranged approximately from -3 to +6 . We focused our initial attention on transcripts expressed at moderate to high levels because they show more prominent dynamics allowing higher confidence in identification of differentially expressed genes. Therefore, 2773 genes whose $\log _{2}$ expression values rose above zero in at least $10 \%$ of all sampled time-points in either strain were considered for initial analysis.
To identify kinetically perturbed genes in YSK4425, we first performed a linear interpolation of the time-series data to obtain corresponding values at every time-point in the two series. We then employed two approaches to identify genes with altered profiles in YSK4425. In the first approach, we calculated a 'difference profile' by subtracting $\log _{2}$ expression value of genes in YSK4425 from corresponding values in M145. These 'difference profiles' were then analyzed using principal component analysis [see Additional file 1]. Two major patterns (Figure 2a) emerged from this analysis, each displaying a significant deviation from zero at different growth stages. Principal component 1 (PC-1) which accounted for $\sim 38 \%$ of all the variance in the 'difference profiles' (Figure $2 \mathrm{~b}$ ) accounted for genes that had progressively higher expression levels in M145 compared to YSK4425 as the culture entered stationary phase. Principal component 2 (PC-2; accounting for $\sim 16 \%$ variation) indicated that some genes also had a tendency to display a higher relative expression in M145 between $25 \mathrm{~h}$ and $30 \mathrm{~h}$ but lower levels thereafter with respect to YSK4425. To identify genes that had extreme values along each of these component axes, we plotted PC-1 versus PC-2 for the selected set moderate/high expression genes (Figure 2c). As we describe in later sections, certain families of genes cluster together in this plot, suggesting coordinate differential regulation for these genes in M145 and YSK4425 
a.

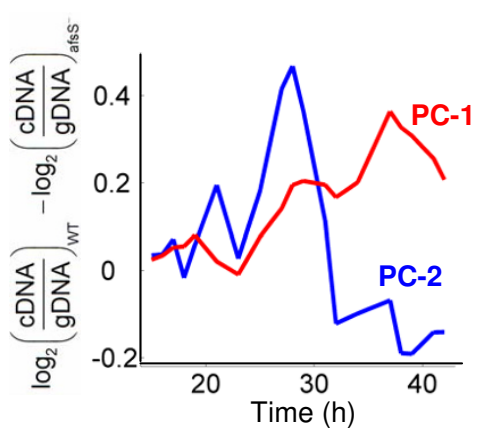

b.

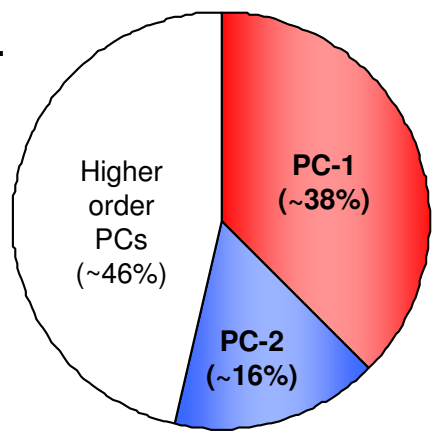

C.

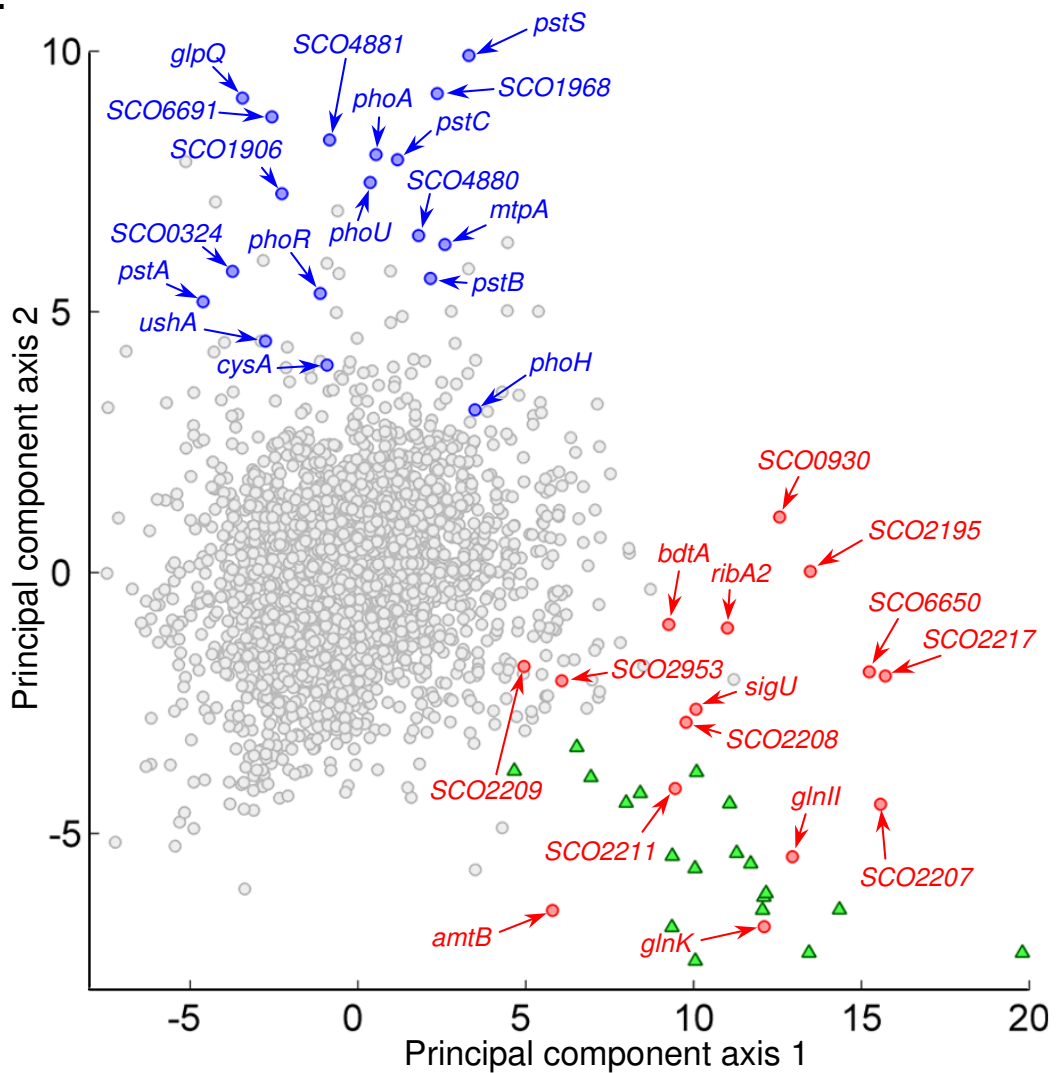

\section{Figure 2}

Identification of patterns among kinetically perturbed genes in YSK4425 using principal component analysis (PCA). (a) Eigenvector plots for principal components I and 2 - PC-I (red) and PC-2 (blue) which represent the major patterns in differential expression profiles. PCA was performed on the 'difference profiles' between the two strains to find patterns in differential expression. (b) Fraction of variation accounted for by the first two and the rest of principal components. (c) Plot of PC-I vs PC-2 identifies genes that have a high value along each of PC-I and PC-2. Actinorhodin biosynthesis genes are shown as filled green triangles, while certain other interesting genes are also marked (red circles for high PC-I and blue circles for high PC-2). The figure indicates that several functionally related genes, particularly phosphate and nitrogen metabolism related elements, and actinorhodin biosynthesis genes cluster together in various regions of the plot.

In a second complementary approach to identify genes with altered profiles in YSK4425, we calculated Euclidean distance between gene profiles in M145 and YSK4425, normalized by the total number of sampling points [see Additional file 1]. Higher Euclidean distances imply that gene expression patterns in the two strains are dissimilar. In general, many genes with high Euclidean distance greater than mean $(\mu)$ plus 1.2 times the standard deviation $(\sigma)$ - also had higher component(s) along PC-1 and/ or PC-2. These Euclidean distance estimates were used as a means to verify findings from principal component analysis and are reported in relevant tables in later sections.

\section{Validation of microarray results using $q R T-P C R$}

Quantitative real-time PCRs were performed on reverse transcribed RNA samples to independently validate the microarray results. A total of 11 genes $($ actIII, redD, redP, phoR, glnII, $g \ln K, c y s D, a b s A 1 / A 2, s c b A$ and $s c b R$ ) were chosen for analysis. Relative expression levels from qRT-PCR for samples $18 \mathrm{~h}, 19 \mathrm{~h}, 21 \mathrm{~h}$ and $38 \mathrm{~h}$ in M145 and $17 \mathrm{~h}$, $19 \mathrm{~h}, 21 \mathrm{~h}$ and $37 \mathrm{~h}$ in YSK4425 with respect to the $18 \mathrm{~h}$ of M145 were plotted against those obtained from microarray experiments (Figure 3). The comparison indicates a good concordance between qRT-PCR and microarray results. We note that the range of dynamics for relative $\log _{2}$ ratios obtained from qRT-PCR $(-4$ to +6$)$ was significantly higher than that from microarray $(-2$ to +5$)$, indicating that qRT-PCRs are more sensitive particularly in low expression ranges. This probably reflects on the Pear- 


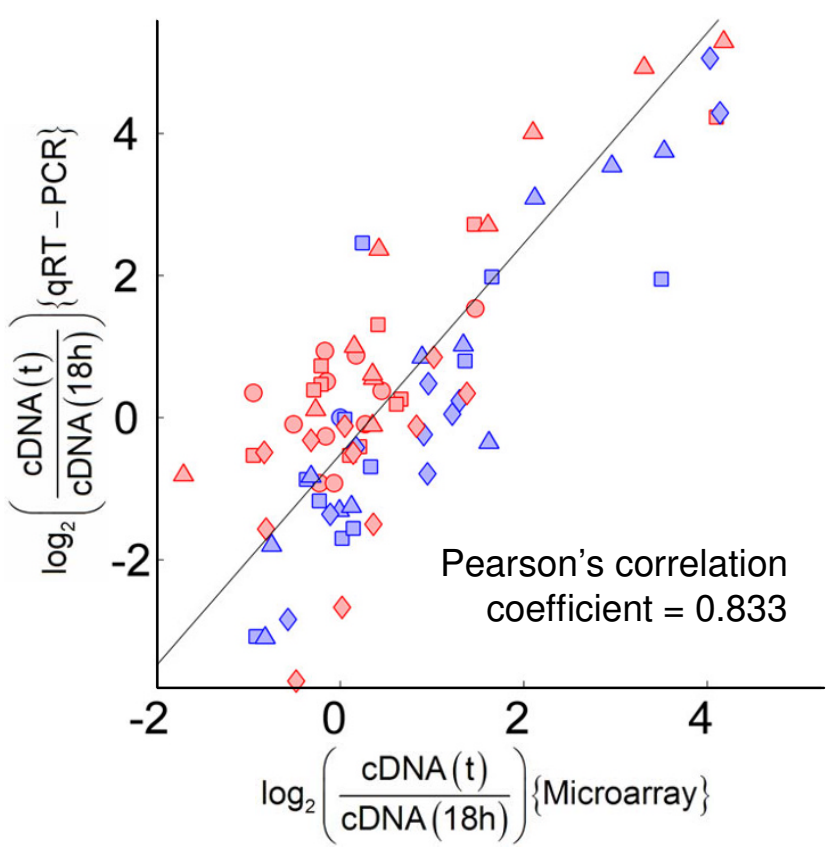

Figure 3

Validation of microarray results using qRT-PCRs. A plot of $\log _{2}$ expression ratios for I I different genes (see text) relative to that from the $18 \mathrm{~h}$ sample of MI 45 culture. Samples from I8 $\mathrm{h}(O), \mathrm{I} 9 \mathrm{~h}(\square), 2 \mathrm{I} \mathrm{h}(\triangle)$ and $38 \mathrm{~h}(\diamond)$ for MI45 are shown in blue while $17 \mathrm{~h}(O), 19 \mathrm{~h}(\square), 2 \mathrm{l} h(\triangle)$ and 37 $\mathrm{h}(\diamond)$ for YSK4425 are shown in red. A least square straight line fit is also shown.

son's correlation coefficient for the plot, resulting in lower value than what could be expected.

\section{Functional classification of genes with altered expression profiles in afsS mutant}

Principal component analysis of microarray data not only identified a set of differentially expressed genes in YSK4425, but also enabled their classification into coordinately perturbed subgroups. Each sub-group then represented a distinct pattern of differential expression; the two most commonly observed patterns were briefly mentioned earlier. Further analysis revealed that genes exhibiting each of these patterns also fell within a handful of functional categories (genes marked in Figure 2c) suggesting a role for AfsS in coordinating those functions. These and other related genes are discussed below with reference to Figure 2c.

\section{Antibiotic biosynthesis genes}

In R5- medium, $S$. coelicolor M145 begins synthesis of the polyketide antibiotic, actinorhodin, nearly $12 \mathrm{~h}$ after entering the stationary phase; antibiotic titers continue to rise in the culture until onset of cell death characterized by gradual fragmentation of mycelia. Microarray analysis revealed that genes belonging to the actinorhodin biosynthesis cluster in M145 were up-regulated slightly ahead of the appearance of blue pigment in culture supernatants. In YSK4425, consistent with the phenotypic observations, we found that these genes were not activated at any stage. By the nature of their profiles in M145 and YSK4425, these genes had a high component along PC-1 discussed earlier (Figure 2c). A direct comparison of the gene expression profiles are shown in Figure 4a. This observation clearly indicates that AfsS exerts its control of actinorhodin synthesis through direct or indirect transcriptional regulation of the relevant biosynthetic genes.

In addition to actinorhodin, at least two other antibiotics, undecylprodigiosin and calcium-dependent antibiotic (CDA) are synthesized by $S$. coelicolor. The expression profiles of both these antibiotic biosynthesis clusters showed a bimodal distribution in M145 with an early peak at 16 $\mathrm{h}$ and a later gradual peak between $24 \mathrm{~h}$ and $32 \mathrm{~h}$. In YSK4425, while the first peak was present at the same time-point as M145, the second peak for many genes were either absent or delayed (Figures $4 \mathrm{~b}$ and $4 \mathrm{c}$ ). These profiles also corroborate with the phenotypic observation that undecylprodigiosin accumulated to a lower extent in YSK4425 compared to M145 particularly in late stationary phase.

\section{Phosphate starvation response and other similarly regulated genes}

Analysis of Figure $2 \mathrm{c}$ revealed that many genes with a high value along PC-2 were either annotated as phosphate starvation response elements or were conspicuously related to phosphate metabolism. These genes also displayed distinct bimodal peaks - at around $20 \mathrm{~h}$ and $27 \mathrm{~h}$ - in M145. A profile search based on correlation coefficient yielded additional genes with similar differential profiles in M145 and YSK4425 (Figure 5a). A total of 56 genes that belonged to this category along with their Euclidean distance values are listed in Table 1 . They include genes encoding the two-component system - phoRP (SCO4229-30), phosphate specific transporters - pstSCAB, alkaline phosphatases (SCO2286, phoA and SCO0324) and glycerophosphoryl diester phosphodiesterase ( $g l p Q)$ homologs (SCO1968 and SCO7550). A large locus (SCO4873-81) consisting of several hypothetical or membrane proteins as well as those involved in putative phosphorus-free teichuronic acid biosynthesis (neuAB) which may substitute for phosphate-rich teichoic acids in cell envelope polymers also belong to this category. A recent study discovered that these genes are PhoP-dependent [23]. In addition, several genes encoding twinarginine translocation (Tat) dependent exported proteins (SCO1196, SCO1565, SCO1633, SCO1906, SCO2286, SCO3790, SCO6691 and SCO7631) identified in an earlier study [24] also belonged to this category. Several other 


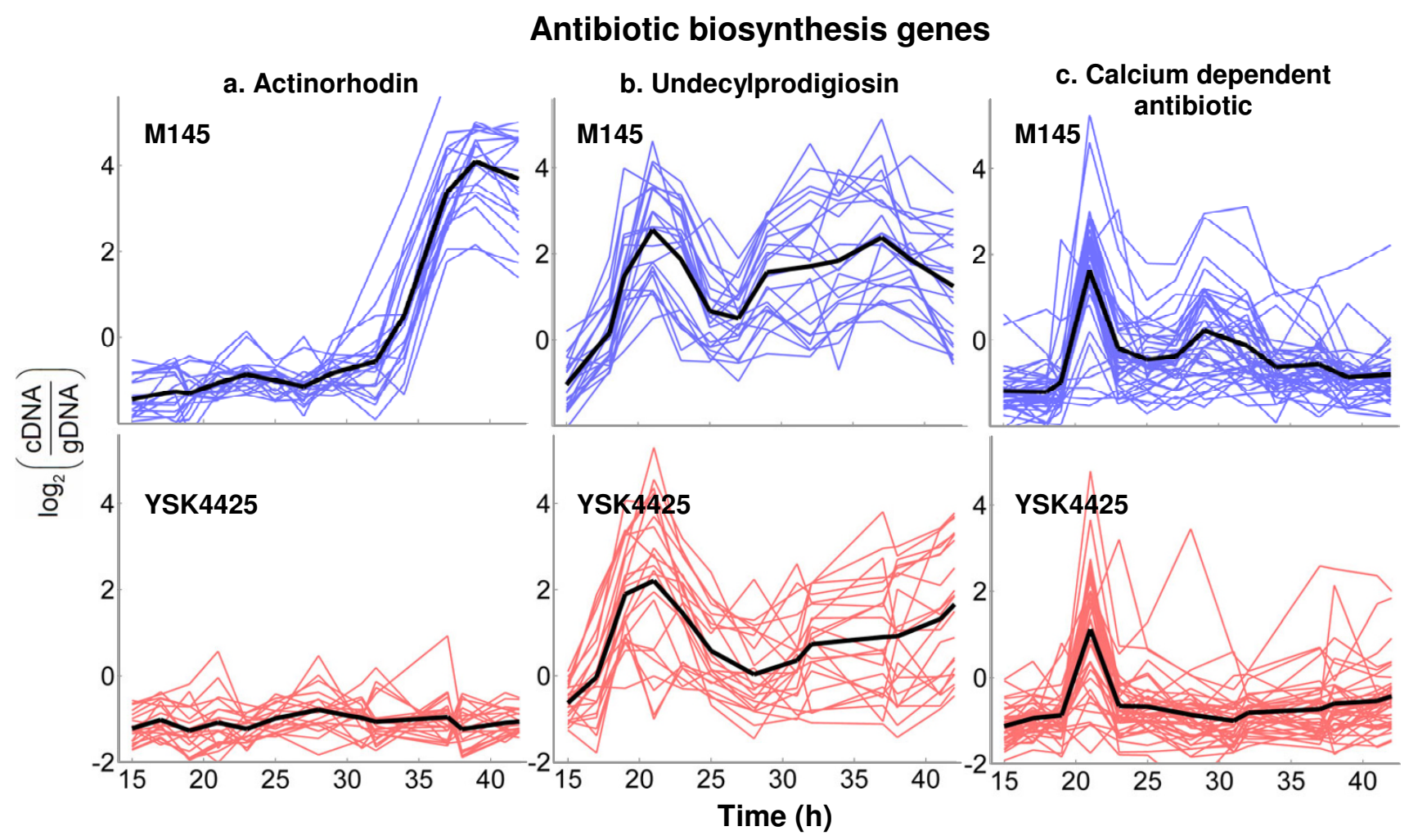

Figure 4

Time profiles representing ' $\log _{2}$ expression value' for antibiotic biosynthesis genes. Profiles from MI 45 (blue) and YSK4425 (red) are shown with the average expression profile in each class emphasized by a thick black line.

genes encoding secreted proteins, nucleases and other hypothetical proteins were also included in this cluster (Table 1). By virtue of their similarity in expression in M145 and differential regulation in YSK4425, we postulate that many of these elements likely belong to the phosphate starvation response system. Transient up-regulation of these genes in M145 might reflect a concerted effort by $S$. coelicolor to counter phosphate starvation by active transport, scavenging, and phosphate regeneration through catabolism from intracellular sources like nucleotides.

It is interesting to note that disruption of afsS led to perturbation of the phosphate starvation response system. Figure 5a indicates that the second of the characteristic bimodal peaks for these genes were either absent or delayed in YSK4425. Earlier studies in S. coelicolor and $S$. lividans using deletion mutants of phoRP [25,26], ppk $[25,27,28]$, and pstS [27] largely concluded that disruption of Pho system components caused a premature phosphate-starvation-like situation leading to overproduction of actinorhodin in these strains. One hypothesis put forward was that phosphorylated PhoP represses transcription of afs $S$ and inhibits actinorhodin synthesis until a time when, perhaps, a more severe form of phosphate starvation or another independent mechanism sets in to relieve this repression [21]. However, contrary to expectations from this hypothesis, we observed here that a delay or inactivation of phosphate starvation response genes in stationary phase was accompanied by a complete abolishment of actinorhodin synthesis. Moreover, although it has been hypothesized that $a f_{s} S$ might be under the control of PhoP, our results indicate that presence of $a f_{s} S$ is required for normal expression of phoRP in stationary phase. Therefore, for the previous hypothesis to be valid, it appears that some form of feedback regulation must exist, whereby AfsS and PhoRP can regulate the transcription of each other.

\section{Nitrogen metabolism genes and their chromosomal neighbors}

The enzyme glutamine synthetase (GS) catalyzes the conversion of $\mathrm{NH}_{4}{ }^{+}$to glutamine, a key step in nitrogen assimilation particularly under low nitrogen conditions. Although, single copies of GS-encoding genes are typically found in most bacteria, the genome of $S$. coelicolor encodes five GS-type enzymes, two of which $(g \ln A$, SCO2198 and glnII, SCO2210) have so far been confirmed 


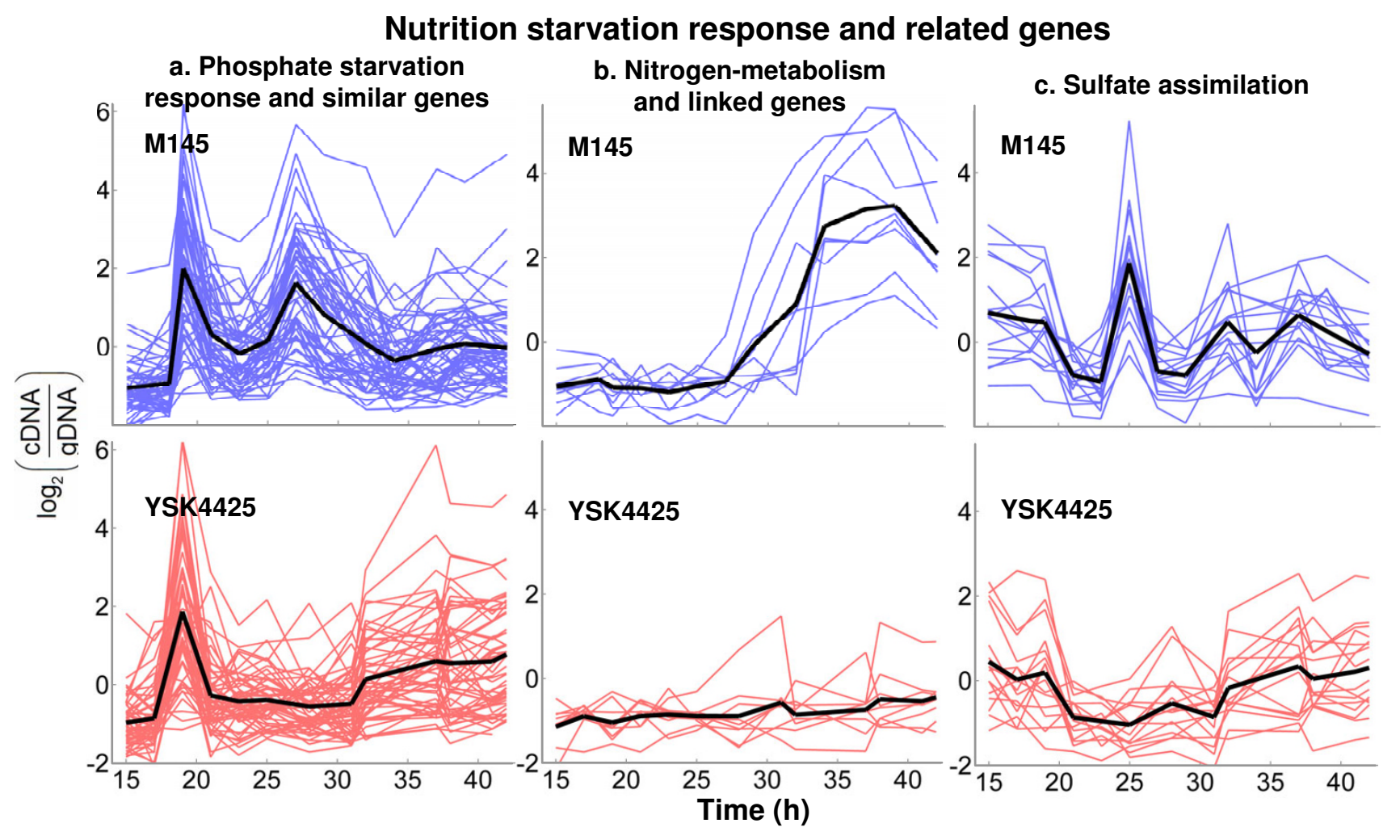

Figure 5

Time profiles representing ' $\log _{2}$ expression value' for nutrient starvation response and related genes. Profiles from MI45 (blue) and YSK4425 (red) are shown with the average expression profile in each class emphasized by a thick black line. The list of genes used in this plot is shown in Table.

to be involved in nitrogen metabolism $[29,30]$. In addition, several other genes, including an ammonium transporter (amtB, SCO5583) and a signal transduction protein PII $(g \ln K, S C O 5584)$ are implicated in nitrogen assimilation $[30,31]$. Our microarray analysis revealed that, consistent with previous observations [32], $g \ln A$, the probable housekeeping glutamine synthetase in $S$. coelicolor was constitutively expressed at high levels in both M145 and YSK4425 (data not shown). However, we found that $g \ln I$, $g \ln K$ and $a m t B$ clustered together with actinorhodin biosynthesis genes in Figure 2c indicating differential expression in strain YSK4425. Like actinorhodin biosynthesis genes, these were activated during stationary phase in M145 but remained dormant throughout the culture period in YSK4425 (Figure 5b). Interestingly, a PhoP-deletion mutant also perturbed the expression of $g \ln I I, g \ln K$ and $a m t B$ indicating a link between phosphate and nitrogen starvation response [23]; but, in contrast to our observations, these genes were up-regulated in the absence of PhoP in those experiments. The effect of glnII expression on antibiotic synthesis is, however, consistent with previous reports. It has been shown previously that a mutation in $r p o B$, the RNA polymerase $\beta$ chain, leads to a remarka- ble increase in glnII expression as well as the induction of actinorhodin biosynthesis in an otherwise non-producing strain of S. lividans [33]. Also, a recent report found that a homolog of S. coelicolor GlnR (the transcriptional regulator of glnA and glnII) in Amycolatopsis mediterranei, when cloned into $S$. coelicolor, had pleiotropic effects on host antibiotics production [34]. These findings suggest that nitrogen starvation response is linked to antibiotic synthesis, and our results propose a central role for AfsS in coordinating this response.

In addition to known nitrogen metabolism genes, certain other chromosomally linked genes also exhibited similar differential profiles in M145 and YSK4425 (Table 1). One of them, SCO2211, located immediately downstream of and co-transcribed with glnII [35], encodes a putative hypothetical protein with a signal peptide sequence. Further downstream, SCO2217 and SCO2218, encoding putative secreted and membrane-bound proteins respectively as well as three upstream genes (SCO2207-09), including a MarR-type transcriptional regulator were similarly regulated. Interestingly, mutational and DNA-binding studies of SCO2208 and SCO2209 [36] concluded that 
Table I: Nutrient metabolism genes with altered expression profiles in YSK4425

\begin{tabular}{|c|c|c|}
\hline Gene & Description & Euclidean Distance ${ }^{\dagger}$ \\
\hline \multicolumn{3}{|c|}{ 1. Phosphate starvation response and other similarly regulated genes } \\
\hline ScOol3I & Endonuclease, exonuclease, phosphatase family protein & 0.16 \\
\hline SCO0324 & Putative secreted alkaline phosphatase & 0.34 \\
\hline SCO0546 & Pyruvate carboxylase (pyc) & 0.13 \\
\hline Sc00919 & Conserved hypothetical protein & 0.15 \\
\hline SCO092I & Conserved hypothetical protein & 0.10 \\
\hline SCO 1048 & Putative secreted protein & 0.32 \\
\hline SCO 1196 & Probable Tat dependent secreted protein & 0.36 \\
\hline SCO 1290 & Putative secreted alkaline phosphatase & 0.19 \\
\hline SCO 1564 & Putative RNA polymerase sigma factor & 0.10 \\
\hline SCO 1565 & Putative glycerophosphoryl diester phosphodiesterase & 0.46 \\
\hline SCOI633 & Tat dependent secreted protein (tat $A)$ & 0.23 \\
\hline SCO1906 & Probable Tat dependent secreted protein & 0.40 \\
\hline SCO 1968 & Glycerophosphoryl diester phosphodiesterase ( $g / p Q)$ & 0.52 \\
\hline SCO 1969 & Putative DNA-methyltransferase & 0.20 \\
\hline SCO2068 & Putative secreted alkaline phosphatase & 0.28 \\
\hline SCO2286 & Putative Tat-dependent secreted alkaline phosphatase $(p h o A)$ & 0.43 \\
\hline SCO2348 & Putative secreted protein & 0.56 \\
\hline SCO2349 & Hypothetical protein, similar to phosphodiesterase (phoD) & 0.15 \\
\hline SCO2532 & PhoH-like protein $(\mathrm{phoH})$ & 0.18 \\
\hline SCO2764 & Putative lipoprotein. & 0.15 \\
\hline SCO2878 & Hypothetical protein & 0.32 \\
\hline SCO3789 & Endonuclease, exonuclease, phosphatase family protein & 0.10 \\
\hline SCO3790 & Conserved hypothetical protein & 0.35 \\
\hline SCO4I39 & Phosphate $A B C$ transport system ATP-binding protein (pstB) & 0.32 \\
\hline SCO4/40 & Phosphate $A B C$ transport system permease protein $(p s t A)$ & 0.36 \\
\hline SCO4I4I & phosphate $A B C$ transport system permease protein (pstC) & 0.41 \\
\hline SCO4I42 & Phosphate-binding protein precursor $(p s t S)$ & 0.57 \\
\hline SCO4/43 & Putative MutT-like protein (mutT) & 0.09 \\
\hline SCO4/44 & Conserved hypothetical protein & 0.20 \\
\hline SCO4I52 & Putative secreted 5'-nucleotidase (ushA) & 0.27 \\
\hline SCO4226 & Hypothetical protein & 0.30 \\
\hline SCO4227 & Metallothionein protein $(m t p A)$ & 0.36 \\
\hline SCO4228 & Phosphate transport system regulatory protein (phoU) & 0.37 \\
\hline SCO 4229 & Sensor kinase $(p h o R)$ & 0.30 \\
\hline SCO 4230 & Response regulator (phoP) & 0.19 \\
\hline SCO440I & Putative lipoprotein & 0.18 \\
\hline SCO4873 & Hypothetical protein & 0.25 \\
\hline SCO4877 & Hypothetical protein & 0.22 \\
\hline SCO4878 & Putative glycosyltransferase & 0.21 \\
\hline SCO4879 & Conserved hypothetical protein & 0.28 \\
\hline SCO4880 & Putative transferase & 0.39 \\
\hline SCO488I & Putative polysaccharide biosynthesis related protein & 0.46 \\
\hline SCO5005 & Hypothetical protein & 0.14 \\
\hline SCO5467 & Muramoyl-pentapeptide carboxypeptidase & 0.09 \\
\hline SCO5746 & Hypothetical protein & 0.22 \\
\hline SCO6I45 & Hypothetical protein & 0.34 \\
\hline SCO669l & Putative phospholipase C & 0.46 \\
\hline SCO7344 & Putative secreted protein & 0.16 \\
\hline SCO7550 & Putative glycerophosphoryl diester phosphodiesterase & 0.29 \\
\hline SCO7631 & Putative secreted protein & 0.24 \\
\hline SCO7697 & Putative secreted hydrolase, phytase (phy) & 0.30 \\
\hline SCO7722 & Conserved hypothetical protein & 0.11 \\
\hline \multicolumn{3}{|c|}{ II. Nitrogen utilization and chromosomally linked genes } \\
\hline SCO2207 & Hypothetical secreted protein & 0.70 \\
\hline SCO2208 & Putative caboxylesterase & 0.45 \\
\hline SCO2209 & Putative transcriptional regulator & 0.26 \\
\hline SCO2210 & Glutamine synthetase $(g \ln l)$ & 0.63 \\
\hline SCO22II & Hypothetical protein & 0.45 \\
\hline
\end{tabular}


Table I: Nutrient metabolism genes with altered expression profiles in YSK4425 (Continued)

\begin{tabular}{|c|c|c|}
\hline SCO2217 & Putative secreted protein & 0.69 \\
\hline $\mathrm{SCO} 2218$ & Putative lipoprotein & 0.21 \\
\hline SCO5583 & Ammonium transporter (amtB) & 0.43 \\
\hline SCO5584 & Nitrogen regulatory protein PII (glnK) & 0.59 \\
\hline \multicolumn{3}{|c|}{ III. Sulfate assimilation genes } \\
\hline SCO2910 & Putative cysteine synthase (cysM) & 0.21 \\
\hline SCO29II & Conserved hypothetical protein & 0.28 \\
\hline SCO29I2 & Hypothetical protein & 0.23 \\
\hline SCO4I64 & Putative thiosulfate sulfurtransferase (cys $A)$ & 0.43 \\
\hline SCO4I65 & Conserved hypothetical protein & 0.32 \\
\hline SCO4293 & Putative threonine synthase (thrC) & 0.15 \\
\hline SCO4294 & Conserved hypothetical protein & 0.20 \\
\hline SCO6094 & Aliphatic sulfonate $A B C$ transporter, permease (ssuC) & 0.10 \\
\hline SCO6095 & Aliphatic sulfonate $A B C$ transporter, binding protein (ssuB) & 0.08 \\
\hline SCO6096 & Aliphatic sulfonate $A B C$ transporter, binding lipoprotein (ssuA) & 0.18 \\
\hline SCO6097 & Sulfate adenylyltransferase subunit I $(c y s N)$ & 0.27 \\
\hline SCO6098 & Sulfate adenylyltransferase subunit $2(c y s D)$ & 0.25 \\
\hline SCO6099 & Adenylylsulfate kinase (cys $C$ ) & 0.19 \\
\hline SCO6 100 & Phosphoadenosine phosphosulfate reductase $(\mathrm{cys} H)$ & 0.21 \\
\hline SCO6101 & Hypothetical protein & 0.21 \\
\hline SCO6102 & Putative nitrite/sulphite reductase (cysl) & 0.25 \\
\hline
\end{tabular}

† Euclidean distance normalized with number of time-points. Compare with the average Euclidean distance $(\mu)$ for all genes $=0.134$ and standard deviation $(\sigma)=0.067$. Euclidean distances $\geq \mu+1.2 \sigma$ indicates significant differential expression. However, there may be other genes which have lower Euclidean distances due to low expression levels. These genes were identified by profile similarity search and difference between MI45 and YSK4425 were confirmed by visual inspection.

these were not involved in nitrogen metabolism. Although it has been shown that deletion of SCO2209 does not affect GS activity in S. coelicolor, one cannot exclude the possibility that these genes are controlled by a common regulator. Our data suggest that transcription of all these genes are either directly or indirectly activated by AfsS activity. It is known that GlnRII (SCO2213) is the exclusive transcriptional activator of glnII while both GlnRII and its homolog, GlnR bind to the promoter of $a m t B-g \ln K-g \ln D$ operon [32]. It is therefore likely that the presence of AfsS induces their expression or, perhaps, enhances their DNA-binding ability in an as yet unknown way.

\section{Sulfate assimilation genes}

A closer inspection of Figure $2 \mathrm{c}$ revealed that a probable thiosulfate sulfurtransferase (SCO4164, cysA) had a reasonably high component along PC-2. Although the 'difference profile' of this gene was similar to many phosphate starvation induced genes discussed earlier, the absolute profile in each of M145 and YSK4425 did not resemble them. Instead the profile was characterized by a sharp but transient up-regulation of $c y s A$ by nearly 60 -fold at $25 \mathrm{~h}$ in M145; the mutant, however, lacked this peak. A genome-wide similarity search yielded 15 additional sulfate assimilation genes each having the same (although not as drastic) distinct pattern in both M145 and YSK4425 (Figure 5c and Table 1). We note that these profiles could be reproduced in an independent biological replicate culture and that a representative profile from this set was verified using qRT-PCR. Several genes organized as possible operons including cysteine biosynthesis enzymes (cysM, cysA, cysK and cysIHCDN) belonged to this category. Certain aliphatic sulfonate assimilation proteins (SCO609496, ssuABC) located immediately downstream of $c y s I H$ $C D N$ also displayed a similar pattern. An earlier study [6] had reported that several of these genes were activated upon osmotic shock and this activation was mediated by a stress response sigma factor, $\sigma^{B}$ (SCO0600). Yet, many other genes reported to be under the control of $\sigma^{B}$ were not affected in YSK4425. Thus it appears that while $\sigma^{B}$ and AfsS may each have a broad set of genes under their control, they probably operate cooperatively in a direct or indirect manner to activate a subset of those genes.

\section{Other differentially regulated genes}

In addition to antibiotic and nutrition starvation genes described above, several other gene profiles were altered in YSK4425. However, these genes might not fit into one of the two major patterns discovered using PCA. Hence, Euclidean distances were used as primary indicators of differential expression to identify additional genes perturbed in YSK4425. Also, unlike earlier cases, not all of the identified genes could be grouped together into functional classes. Therefore, in the absence of additional confidence through similar behavior from functionally related genes, we resorted to a stricter statistical test based on significance analysis of time-series data using profiles from biological replicate cultures (see Methods and [37,38]). This analysis provides a $q$-value for every gene, an estimate of false discovery rate when all genes with lower $q$-values are called as differentially expressed. A $q$-value less than 0.25 
was taken as an indication of significant differential expression. We note that at this confidence, the $p$-value for expression profiles were less than 0.008 . These calculations were performed in addition to the Euclidean distance estimates described earlier. Genes that passed the Euclidean distance cutoff $(\geq \mu+1.2 \sigma)$ in both replicate cultures as well as a $q$-value cutoff $(\leq 0.25)$ from the significance analysis test were further inspected manually and reported in Table 2.

As with most cases discussed earlier, the effect of afs $S$ mutation was not visible until mid/late stationary phase for many of these genes. Among them is a major $S$. coelicolor catalase (SCO0379, catA) which was up-regulated by nearly eight-fold in M145 during stationary phase but displayed a less prominent increase in YSK4425. A few genes downstream, SCO0382 and SCO0383, which are categorized as secondary metabolism genes (deoxysugar synthase cluster) displayed a similar pattern. In fact, several genes in the deoxysugar synthase/glycosyl transferase cluster (SCO0381-401) were similarly regulated (data not shown) although many did not meet our statistical criteria due to smaller fold-changes. The stationary phase activation of another set of genes (SCO1627-30, rarABCD) organized as a single operon was also attenuated in YSK4425. These genes have previously been implicated in control of glucose-dependent activation of actinorhodin biosynthesis in S. coelicolor [39]. Interestingly, carbon source dependent modulation of $a f_{s} S$ has been shown earlier to be responsible for activation of antibiotic synthesis in S. lividans [40]. Two additional proteins, SCO2195-96, encoding a hypothetical and membrane bound protein respectively were also significantly suppressed in YSK4425 during stationary phase.

The "sigma-like" domains of AfsS do not contain any obvious DNA-binding sites. Yet, as described here, a large set of genes with diverse functions appear to be under its control. This prompted us to search for possible transcriptional regulators differentially expressed in YSK4425 as these could be the potential mediators through which AfsS may exert its control on downstream targets. Our analysis indicated that expression profile of a putative ECF-sigma factor (SCO5147) was significantly altered in YSK4425 (Table 2). Consistent with this finding, a recent study had identified this gene as a positive regulator of actinorhodin synthesis in S. coelicolor [41]. Moreover, it was also found that activation of SCO5147 accompanied the up-regulation of $a f s S$ in a redZ over-expressing strain [42]. The expression profiles of another putative regulator, SCO7252 and its upstream neighbor, SCO7251, were also dramatically altered in YSK4425. Although these genes have not been previously studied in detail, it is known that SCO7252 is homologous to a negative regulator of differentiation and antibiotic synthesis, nsdA, in S. coelicolor [43]. In addition to these regulators, we note that another sigma factor (SCO2954, sigU) and its cognate anti-sigma factor (SCO2953, rsuA) were both suppressed in one of the replicate cultures in YSK4425 during stationary phase (transcript levels nearly eight-fold higher in M145 compared to YSK4425); the difference in the other culture, though, was not as drastic. However, this observation might still be interesting because an rsuA deletion strain was significantly delayed in actinorhodin synthesis in S. coelicolor [3]. We also note that sigU as well as

Table 2: Differentially expressed regulatory genes identified using Euclidean distance and significance analysis test

\begin{tabular}{|c|c|c|c|}
\hline Gene & Description & Euclidean Distancet & q-value* \\
\hline SCO0379 & Catalase $(k a t A)$ & 0.38 & 0.011 \\
\hline SCO038I & Putative glycosyl transferase & 0.20 & 0.232 \\
\hline SCO0382 & UDP-glucose/GDP-mannose family dehydrogenase (ugd) & 0.30 & 0.0038 \\
\hline SCO0383 & Hypothetical protein & 0.28 & 0.035 \\
\hline SCO0736 & Putative secreted protein & 0.33 & 0.022 \\
\hline SCO0930 & Putative lipoprotein & 0.59 & 0.123 \\
\hline SCOI627 & Putative ATP-GTP binding protein ( $r a r D)$ & 0.39 & 0.221 \\
\hline SCO 1629 & Conserved hypothetical protein (rarB) & 0.39 & 0.116 \\
\hline SCO 1630 & Putative integral membrane protein (rarA) & 0.35 & 0.144 \\
\hline $\mathrm{SCO} 2195$ & Hypothetical protein & 0.59 & 0.236 \\
\hline SCO2196 & Putative integral membrane protein & 0.33 & 0.053 \\
\hline SCO2953 & Putative anti sigma factor $(r s u A)$ & 0.31 & 0.296 \\
\hline SCO2954 & Putative RNA polymerase sigma factor (sigU) & 0.46 & 0.412 \\
\hline SCO $5 / 47$ & putative ECF-subfamily sigma factor (sigE) & 0.31 & 0.044 \\
\hline SCO725I & Conserved hypothetical protein & 0.30 & 0.169 \\
\hline SCO7252 & Putative regulatory protein & 0.37 & 0.0038 \\
\hline
\end{tabular}

† Euclidean distance normalized with number of time-points. Compare with the average Euclidean distance $(\mu)$ for all genes $=0.134$ and standard deviation $(\sigma)=0.067$. Euclidean distances $\geq \mu+1.2 \sigma$ indicates significant differential expression.

*q-value from significance analysis of time-series data. Values less than 0.25 are statistically significant. 
SCO7251 and SCO7252 were recently reported as differentially expressed in a PhoP-deletion strain [23].

\section{Conclusion}

AfsS-like proteins are relatively rare in nature. Thus far, only a handful of related streptomycetes like S. lividans, $S$. griseus and $S$. noursei are known to encode such proteins. With no precedent analyses from model organisms like Escherichia coli and Bacillus subtilis or closer relatives like Corynebacteria and Mycobacteria, it should, perhaps, come as no surprise that even the basic molecular function of AfsS has not been elucidated yet. In this work, we have demonstrated that AfsS regulates both antibiotic synthesis and nutrition starvation response genes in $S$. coelicolor. Over 117 genes were perturbed in the afs disruption strain. An overwhelming majority of these genes were down-regulated in the mutant strain indicating that AfsS is primarily a direct or indirect positive regulator of transcription for numerous downstream genes. A common thread linking almost all these observations was that the phenotypic and transcriptional effects of the mutation were not evident until the onset of stationary phase. In this aspect, AfsS resembles alterative sigma factors, many of which have been implicated in stress response and stationary phase adaptation in bacteria. In fact, AfsS is annotated as a "sigma-like" protein because it contains certain conserved residues of the domain 3 of sigma factors [44]. This domain, containing three $\alpha$-helices, mainly interacts with the $\beta$-subunit of RNA polymerase and acts as a linker between domains 2 and 4 [4]. Interestingly, ECF sigma factors, 51 of which are found in S. coelicolor, lack this domain and instead contain only a short stretch of linker segment. Thus, an intriguing possibility that may, perhaps, be tested in future studies is if AfsS acts cooperatively with one or more of $S$. coelicolor ECF sigma factors.

One possible hypothesis that can be put forth from this work is that the regulation of actinorhodin synthesis by AfsS is mediated by phosphate starvation genes. Thus, in the absence of AfsS, the phosphate starvation mechanism is impaired leading to the abolishment of actinorhodin synthesis. An alternative hypothesis would suggest that AfsS activates actinorhodin synthesis in an as yet unknown manner, but, under the conditions tested, antibiotic biosynthesis requires sequestering of cellular resources through expression of phosphate, sulfate and nitrogen metabolism genes. Thus, abolishment of actinorhodin synthesis by disruption of afsS obviates the need for expression of these genes. A third possible hypothesis would be that the activation of nutrient starvation genes and antibiotic biosynthesis genes are two unrelated activities of AfsS. Further work is needed to test these hypotheses. Irrespective of these possibilities and the mode of action of AfsS, we have shown that it is a master regulator of both antibiotic synthesis and nutrition starvation response genes in S. coelicolor. The findings reported here should provide some important clues to unraveling the intricate antibiotic regulatory machinery in $S$. coelicolor and related microbes.

\section{Methods \\ Bacterial strains, growth conditions and assays}

S. coelicolor A3(2) strains M145 and YSK4425 ( $\triangle a$ afsS::apr) were cultured in R5 agar [45] for generation of high concentration spore suspensions. For liquid culture, spores were germinated in 2xYT medium [45] and inoculated into R5- liquid medium [20] lacking sucrose at a density of $\sim 10^{7}$ spores $/ \mathrm{ml}$. Baffled 2 L flasks containing stainless steel coils with $350 \mathrm{ml}$ working volume were used for liquid cultures. Cultures were incubated at $30^{\circ} \mathrm{C}$ in an orbital shaker at $300 \mathrm{rpm}$ and cell growth was monitored by measuring optical density at $450 \mathrm{~nm}$. Antibiotic assays were generally carried out as described elsewhere [45]. Since the amounts of intracellular actinorhodin produced was much less than the extracellular $\gamma$-actinorhodin under the conditions tested, only data for $\gamma$-actinorhodin are shown in the results.

\section{Disruption of afsS and complementation}

The afsS disruption mutant in S. coelicolor M145 was constructed by replacing a major segment of the afs $S$ coding region with an apramycin resistance cassette (apr). This was achieved by first constructing a disruption plasmid by four-way ligation of a host vector backbone (pDHS901, a derivative of pGM160 E. coli-Streptomyces shuttle vector) bearing the thiostrepton resistance gene (tsr), a $1 \mathrm{~kb}$ fragment identical to the left flanking region of the targeted gene, the apr cassette and a $1 \mathrm{~kb}$ fragment identical to the right flanking region of the targeted gene. The primers used to amplify the target flanking regions were $5^{\prime}$ GCAAGCTTAGGGCTCGCACCGTTCTCAGC-3' and 5'GCGAATTCGTCCGCGTCCTTCATCTTGTC-3' for the left segment and 5'-GCCTGCAGACCACGATGGACAACCACATG-3' and 5'-GCGGATCCTACACCCTGGACGCGGTCACC-3' for the right segment. This disruption plasmid was propagated in the methylation deficient $E$. coli host ET12567 and subsequently transformed into S. coelicolor M145 through protoplast transformation. Plasmid uptake and homologous recombination was selected for by choosing colonies resistant to apramycin. A subsequent screen for thiostrepton sensitive strains yielded cells that underwent double crossover recombination. Southern hybridizations and PCR were used to confirm the disruption of afsS.

Mutants were complemented by reintroduction of the wild-type afsS allele. For this, pSET152-kan, a modified version of pSET152 (with a S. coelicolor bacteriophage $\phi C 31$ recombination site attB [46]) was first constructed by inserting a pUC-NEO [47] derived kanamycin resist- 
ance gene into its PacI site. An 1192 bp PCR amplified DNA segment comprising 500 bp upstream of start codon (to include the promoter), the wild-type afs $S$ gene and 500 bp downstream of stop codon (to include any possible transcription terminators) was then cloned into the EcoRI-XbaI site of pSET152-kan. The resulting plasmid was transferred into $S$. coelicolor by conjugation via a nonmethylating E. coli donor ET12567 containing pUZ8002 [45]. Reintroduction of the wild-type afs $S$ gene in the exconjugant was confirmed by PCR and subsequent sequencing.

\section{RNA extraction}

Immediately after harvesting culture extracts, a one-fifth volume of an ice-cold stop solution (5\% phenol in ethanol) was added to prevent RNA degradation [48]. The sample was quickly centrifuged at $4{ }^{\circ} \mathrm{C}$ and cell pellets were stored at $-80^{\circ} \mathrm{C}$ until RNA extraction. To extract RNA, the frozen cell pellets were lyophilized using liquid $\mathrm{N}_{2}$ and resuspended in buffer RLT (RNeasy Mini Kit, Qiagen Inc., Valencia, CA). Further steps in RNA extraction were carried out in accordance with the manufacturer's instructions. RNA integrity was determined by gel electrophoresis while the quantity and purity was estimated by absorbance at $260 \mathrm{~nm}$ and $280 \mathrm{~nm}$.

\section{Microarray hybridizations}

S. coelicolor microarray chips reported earlier [22] were used. $10 \mu \mathrm{g}$ of total RNA and $200 \mathrm{ng}$ genomic DNA (gDNA) were used in all hybridizations. Total RNA was reverse transcribed to cDNA by SuperscriptII (Invitrogen, Carlsbad, CA) with random hexamer primers (IDT, Coralville, IA) with concomitant incorporation of aminoallyldUTP (Ambion, Austin, TX). cDNA was then incubated with Alexa 647 (Invitrogen, Carlsbad, CA) for labeling. Fragmented $S$. coelicolor gDNA was chemically labeled using Label $\mathrm{IT}^{\circledast} \mathrm{Cy} 3$ reagents (Mirus Bio Corp., Madison, WI). The labeled cDNA and gDNA were then mixed and co-hybridized to microarray slides in the presence of $50 \%$ formamide. After 16 hours of incubation at $50^{\circ} \mathrm{C}$, slides were washed and scanned with ScanArray5000 (Perkin Elmer, Wellesley, MA). Details of all protocols are available online [49].

GenePix (Molecular Devices, Union City, CA) was used to process scanned images and obtain raw intensity data for each spot. The median fluorescence intensity from each channel was used to calculate a $\log _{2}$ ratio of cDNA/gDNA. A quantile normalization strategy [22] was adopted to normalize data from multiple chips. The resulting $\log _{2}$ ratios were both used to compare transcript levels of the same mRNA in different samples as well as to estimate the relative abundance levels of different mRNA in a single sample.

\section{Quantitative real-time PCRs}

Reverse transcription of total RNA was performed on selected samples with $1 \mu \mathrm{g}$ of total RNA and $1 \mu \mathrm{g}$ of random hexamer primer (Amersham Biosciences, Piscataway, NJ) using Superscript ${ }^{\mathrm{TM}}$ II RNase $\mathrm{H}^{-}$Reverse Transcriptase (Invitrogen). FullVelocity ${ }^{\mathrm{TM}} \mathrm{SYBR}^{\circledR}$ Green QPCR kit (Stratagene, La Jolla, CA) was used for real-time quantitative PCRs on Mx3005P (Stratagene). Reactions were carried out in triplicate and appropriate controls were included to verify the absence of gDNA contamination in RNA, and primer-dimer formation. A standard threshold of 0.2 was used on the ROX-normalized $\log$ (fluorescence) $v$ s. cycle number chart (amplification plot) as a cut-off for $\mathrm{C}_{t}$ (threshold cycle number). $\mathrm{C}_{t}$ values were normalized with respect to SCO5820 ( $h r d B)$, the major vegetative sigma factor of $S$. coelicolor. Relative changes in gene expression were quantified using $2^{-\Delta \Delta \mathrm{Ct}}$ method [50] with the 18 h sample from M145 being chosen as the control sample.

\section{Time-series transcript profile analysis}

Certain inherent difficulties associated with liquid cultures of $S$. coelicolor like pellet formation make accurate estimation of growth, and therefore alignment of growth stages between strains, using optical density measurements difficult. Hence microarray-derived global gene expression patterns were used to align wild-type and mutant cultures using a time-warp strategy reported earlier $[22,51]$. This method aligns the time-frames of different cultures by minimizing the global average Euclidean distance of all gene expression profile pairs. The premise behind this manipulation is that the vast majority of genes are not kinetically differentially expressed between the wild-type and mutant and that only a small set of genes exhibit any differential expression. For cultures performed in the current study, time-warp results suggested an approximate linear shift of adding $5 \mathrm{~h}$ for afs $S$ mutant profiles with respect to the wild-type. This time-aligned data is reported throughout this manuscript.

Euclidean distance estimates and principal component analysis of the resulting time-series data were used to detect differences between expression profiles of genes in wild-type and mutant. For genes that could not be grouped into coordinately perturbed functional classes, a further statistical assessment was performed through significance analysis of time-series data using EDGE $[37,38]$. This method, which requires replicate time-series experiments, is based on a statistical framework and uses polynomial regression to account for the temporal nature of the data. For each gene, the null hypothesis was tested by comparing the coefficients of the polynomial fits from the two groups (M145 and YSK4425). 


\section{Microarray data availability}

Microarray data used in this study has been made available at NCBI - Gene Expression Omnibus [52] in a MIAME-compliant manner: series accession numbers GSE8107 (M145) and GSE8160 (YSK4425).

\section{Authors' contributions}

WL and KPJ carried out liquid culture experiments, printed DNA microarrays, performed transcriptome experiments, overall data analysis, interpretations and manuscript writing. SC performed qRT-PCR verifications and statistical analysis. SM participated in microarray design and assisted in preliminary data analysis. FG helped with microarray construction and liquid culture experiments. YSK created the mutant strain used in this work. DHS and WSH supervised the study, reviewed results and assisted in manuscript preparation. All authors have read and approved the final manuscript.

\section{Additional material}

\section{Additional file 1}

Table of PC-1, PC-2, Euclidean distance and q-values. Complete list of PC-1,PC-2, Euclidean distance and q-value estimates for all the genes are provided in one worksheet. The set of genes passing the Euclidean distance and statistical test cut-offs are shown in a separate worksheet.

Click here for file

[http://www.biomedcentral.com/content/supplementary/14712164-9-56-S1.xls]

\section{Acknowledgements}

We thank Siguang Sui for his assistance with mutant complementation experiments performed in this study. This work was supported in part by a grant from the National Institutes of Health GM55850. The bioinformatics support was provided by Minnesota Supercomputing Institute at the University of Minnesota. Microarray printing and imaging support was provided by Biomedical Image Processing Laboratory at the University of Minnesota. SM was supported by a Graduate School Fellowship from the University of Minnesota.

\section{References}

I. Challis GL, Hopwood DA: Synergy and contingency as driving forces for the evolution of multiple secondary metabolite production by Streptomyces species. Proc Natl Acad Sci USA 2003, I00(Suppl 2): | 4555-| 456 I.

2. Cho YH, Lee EJ, Ahn BE, Roe JH: SigB, an RNA polymerase sigma factor required for osmoprotection and proper differentiation of Streptomyces coelicolor. Mol Microbiol 200I, 42:205-2I4.

3. Gehring AM, Yoo NJ, Losick R: RNA polymerase sigma factor that blocks morphological differentiation by Streptomyces coelicolor. J Bacteriol 200I, 183:599|-5996.

4. Gruber TM, Gross CA: Multiple sigma subunits and the partitioning of bacterial transcription space. Annu Rev Microbiol 2003, 57:44I-466.

5. Lee EJ, Cho YH, Kim HS, Ahn BE, Roe JH: Regulation of $\sigma^{\mathbf{B}}$ by an anti- and an anti-anti-sigma factor in Streptomyces coelicolor in response to osmotic stress. / Bacteriol 2004, I 86:8490-8498.

6. Lee EJ, Karoonuthaisiri N, Kim HS, Park JH, Cha CJ, Kao CM, Roe JH: A master regulator sigma governs osmotic and oxidative response as well as differentiation via a network of sigma factors in Streptomyces coelicolor. Mol Microbiol 2005, 57:1252-1264.

7. Paget MS, Chamberlin L, Atrih A, Foster SJ, Buttner MJ: Evidence that the extracytoplasmic function sigma factor $\sigma^{\mathrm{E}}$ is required for normal cell wall structure in Streptomyces coelicolor A3(2). J Bacteriol |999, I 8 I:204-2 I I.

8. Sevcikova B, Kormanec J: Differential production of two antibiotics of Streptomyces coelicolor A3(2), actinorhodin and undecylprodigiosin, upon salt stress conditions. Arch Microbiol 2004, I 81 :384-389.

9. Bystrykh L, Fernandez-Moreno M, Herrema J, Malpartida F, Hopwood $D$, Dijkhuizen L: Production of actinorhodin-related "blue pigments" by Streptomyces coelicolor A3(2). J Bacteriol 1996, 1 78:2238-2244.

10. Doull JL, Vining LC: Nutritional control of actinorhodin production by Streptomyces coelicolor A3(2): suppressive effects of nitrogen and phosphate. Appl Microbiol Biotechnol 1990, 32:449-454.

II. Bentley SD, Chater KF, Cerdeno-Tarraga AM, Challis GL, Thomson NR, James KD, Harris DE, Quail MA, Kieser H, Harper D, et al.: Complete genome sequence of the model actinomycete Streptomyces coelicolor A3(2). Nature 2002, 417:|4|-|47.

12. Horinouchi S: AfsR as an integrator of signals that are sensed by multiple serine/threonine kinases in Streptomyces coelicolor A3(2). I Ind Microbiol Biotechnol 2003, 30:462-467.

13. Umeyama T, Lee PC, Horinouchi S: Protein serine/threonine kinases in signal transduction for secondary metabolism and morphogenesis in Streptomyces. Appl Microbiol Biotechnol 2002, 59:419-425.

14. Lee Y, Kim K, Suh JW, Rhee S, Lim Y: Binding study of AfsK, a Serl Thr kinase from Streptomyces coelicolor A3(2) and S-adenosyl-L-methionine. FEMS Microbiol Lett 2007, 266:236-240.

15. Umeyama T, Horinouchi S: Autophosphorylation of a bacterial serine/threonine kinase, AfsK, is inhibited by KbpA, an AfsKbinding protein. J Bacteriol 2001, 183:5506-55 I2.

16. Sawai R, Suzuki A, Takano Y, Lee PC, Horinouchi S: Phosphorylation of AfsR by multiple serine/threonine kinases in Streptomyces coelicolor A3(2). Gene 2004, 334:53-6I.

17. Vogtli M, Chang PC, Cohen SN: afsR2: a previously undetected gene encoding a 63-amino-acid protein that stimulates antibiotic production in Streptomyces lividans. Mol Microbiol 1994, 14:643-653.

18. Floriano $B$, Bibb $M: a f s R$ is a pleiotropic but conditionally required regulatory gene for antibiotic production in Streptomyces coelicolor A3(2). Mol Microbiol 1996, 21 :385-396.

19. Lee PC, Umeyama T, Horinouchi S: afsS is a target of AfsR, a transcriptional factor with ATPase activity that globally controls secondary metabolism in Streptomyces coelicolor A3(2). Mol Microbiol 2002, 43: $1413-1430$.

20. Huang J, Lih CJ, Pan KH, Cohen SN: Global analysis of growth phase responsive gene expression and regulation of antibiotic biosynthetic pathways in Streptomyces coelicolor using DNA microarrays. Genes Dev 200I, I5:3 I83-3192.

21. Martin JF: Phosphate control of the biosynthesis of antibiotics and other secondary metabolites is mediated by the PhoRPhoP system: an unfinished story. J Bacteriol 2004, I86:5197-5201.

22. Mehra S, Lian W, Jayapal KP, Charaniya SP, Sherman DH, Hu WS: A framework to analyze multiple time series data: a case study with Streptomyces coelicolor. J Ind Microbiol Biotechnol 2006, 33:159-172.

23. Rodriguez-Garcia A, Barreiro C, Santos-Beneit F, Sola-Landa A, Martin JF: Genome-wide transcriptomic and proteomic analysis of the primary response to phosphate limitation in Streptomyces coelicolor MI45 and in a $\triangle$ phoP mutant. Proteomics 2007, 7:2410-2429.

24. Widdick DA, Dilks K, Chandra G, Bottrill A, Naldrett M, Pohlschro$\operatorname{der} \mathrm{M}$, Palmer T: The twin-arginine translocation pathway is a major route of protein export in Streptomyces coelicolor. Proc Natl Acad Sci USA 2006, 103:17927-17932.

25. Ghorbel S, Kormanec J, Artus A, Virolle M]: Transcriptional studies and regulatory interactions between the phoR-phoP operon and the phoU, mtpA, and ppk genes of Streptomyces lividans TK24. J Bacteriol 2006, 1 88:677-686. 
26. Sola-Landa A, Moura RS, Martin JF: The two-component PhoRPhoP system controls both primary metabolism and secondary metabolite biosynthesis in Streptomyces lividans. Proc Natl Acad Sci USA 2003, 100:6133-6138.

27. Diaz M, Esteban A, Fernandez-Abalos JM, Santamaria RI: The highaffinity phosphate-binding protein PstS is accumulated under high fructose concentrations and mutation of the corresponding gene affects differentiation in Streptomyces lividans. Microbiology 2005, I 5 I :2583-2592.

28. Ghorbel S, Smirnov A, Chouayekh H, Sperandio B, Esnault C, Kormanec J, Virolle MJ: Regulation of ppk expression and in vivo function of Ppk in Streptomyces lividans TK24. J Bacteriol 2006, I 88:6269-6276.

29. Fink D, Falke D, Wohlleben W, Engels A: Nitrogen metabolism in Streptomyces coelicolor A3(2): modification of glutamine synthetase I by an adenylyltransferase. Microbiology 1999, I45(Pt 9):2313-2322.

30. Reuther J, Wohlleben W: Nitrogen metabolism in Streptomyces coelicolor: transcriptional and post-translational regulation. J Mol Microbiol Biotechnol 2007, I 2: | 39-|46.

31. Hesketh A, Fink D, Gust B, Rexer HU, Scheel B, Chater K, Wohlleben W, Engels A: The GInD and GInK homologues of Streptomyces coelicolor A3(2) are functionally dissimilar to their nitrogen regulatory system counterparts from enteric bacteria. Mol Microbiol 2002, 46:319-330.

32. Fink D, Weissschuh N, Reuther J, Wohlleben W, Engels A: Two transcriptional regulators $G \ln R$ and $G \ln R I I$ are involved in regulation of nitrogen metabolism in Streptomyces coelicolor A3(2). Mol Microbiol 2002, 46:331-347.

33. Lai C, Xu J, Tozawa Y, Okamoto-Hosoya Y, Yao X, Ochi K: Genetic and physiological characterization of rpoB mutations that activate antibiotic production in Streptomyces lividans. Microbiology 2002, I 48:3365-3373.

34. $\mathrm{Yu} \mathrm{H}$, Yao $Y$, Liu $Y$, Jiao $R$, Jiang $W$, Zhao GP: A complex role of Amycolatopsis mediterranei GInR in nitrogen metabolism and related antibiotics production. Arch Microbiol 2007, I 88:89-96.

35. Charaniya S, Mehra S, Lian W, Jayapal KP, Karypis G, Hu WS: Transcriptome dynamics-based operon prediction and verification in Streptomyces coelicolor. Nucleic Acids Res 2007.

36. Weisschuh N, Fink D, Vierling S, Bibb MJ, Wohlleben W, Engels A Transcriptional analysis of the gene for glutamine synthetase II and two upstream genes in Streptomyces coelicolor A3(2). Mol Gen Genet 2000, 264:46 I-469.

37. Leek JT, Monsen E, Dabney AR, Storey JD: EDGE: extraction and analysis of differential gene expression. Bioinformatics 2006, 22:507-508

38. Storey JD, Xiao W, Leek JT, Tompkins RG, Davis RW: Significance analysis of time course microarray experiments. Proc Nat Acad Sci USA 2005, 102: I 2837- 12842.

39. Komatsu M, Kuwahara Y, Hiroishi A, Hosono K, Beppu T, Ueda K Cloning of the conserved regulatory operon by its aerial mycelium-inducing activity in an amfR mutant of Streptomyces griseus. Gene 2003, 306:79-89.

40. Kim ES, Hong HJ, Choi CY, Cohen SN: Modulation of actinorhodin biosynthesis in Streptomyces lividans by glucose repression of afsR2 gene transcription. J Bacteriol 200I, I 83:2 I 98-2203.

4I. Kang SH, Huang J, Lee HN, Hur YA, Cohen SN, Kim ES: Interspecies DNA microarray analysis identifies WbIA as a pleiotropic down-regulator of antibiotic biosynthesis in Streptomyces. J Bacteriol 2007, 189:4315-4319.

42. Huang J, Shi J, Molle V, Sohlberg B, Weaver D, Bibb MJ, Karoonuthaisiri N, Lih CJ, Kao CM, Buttner MJ, Cohen SN: Cross-regulation among disparate antibiotic biosynthetic pathways of Streptomyces coelicolor. Mol Microbiol 2005, 58:1276- 1287.

43. Li W, Ying X, Guo Y, Yu Z, Zhou X, Deng Z, Kieser H, Chater KF, Tao $M$ : Identification of a gene negatively affecting antibiotic production and morphological differentiation in Streptomyces coelicolor A3(2). J Bacteriol 2006, I 88:8368-8375.

44. Kim CY, Park HJ, Kim ES: Functional dissection of sigma-like domain in antibiotic regulatory gene, afs $R 2$ in Streptomyces lividans. J Microbiol Biotechnol 2006, 16:1477-|480.

45. Kieser T, Bibb MJ, Buttner MJ, Chater KF, Hopwood DA: Practical Streptomyces Genetics Norwich, United Kingdom: The John Innes Foundation; 2000.
46. Bierman M, Logan R, O'Brien K, Seno ET, Rao RN, Schoner BE: Plasmid cloning vectors for the conjugal transfer of DNA from Escherichia coli to Streptomyces spp. Gene 1992, I I 6:43-49.

47. Williamson JM, Inamine E, Wilson KE, Douglas AW, Liesch JM, Albers-Schonberg $\mathrm{G}$ : Biosynthesis of the $\beta$-lactam antibiotic, thienamycin, by Streptomyces cattleya. Journal of Biological Chemistry 1985, 260:4637-4647.

48. Bernstein JA, Khodursky AB, Lin PH, Lin-Chao S, Cohen SN: Global analysis of mRNA decay and abundance in Escherichia coli at single-gene resolution using two-color fluorescent DNA microarrays. Proc Natl Acad Sci USA 2002, 99:9697-9702.

49. Streptomyces coelicolor DNA microarray protocols [https:// hugroup.cems.umn.edu/Protocols/protocol.htm]

50. Livak KJ, Schmittgen TD: Analysis of relative gene expression data using real-time quantitative PCR and the $\mathbf{2}^{-\Delta \Delta \mathrm{Ct}}$ method. Methods 200I, 25:402-408.

51. Aach J, Church GM: Aligning gene expression time series with time warping algorithms. Bioinformatics 200I, I 7:495-508.

52. Gene Expression Omnibus [http://www.ncbi.nlm.nih.gov/geo/]
Publish with Biomed Central and every scientist can read your work free of charge

"BioMed Central will be the most significant development for disseminating the results of biomedical research in our lifetime. "

Sir Paul Nurse, Cancer Research UK

Your research papers will be:

- available free of charge to the entire biomedical community

- peer reviewed and published immediately upon acceptance

- cited in PubMed and archived on PubMed Central

- yours - you keep the copyright

Submit your manuscript here:

http://www.biomedcentral.com/info/publishing_adv.asp
BioMedcentral 